\title{
Nicht einmal im Mutterleib sicher
}

W issenschaftler verfolgen ihre Suche nach pathogenetischen Ursachen atopischer Erkrankungen bis in die frühsten Lebensmonate. Ihre Hypothese lautet: Ereignisse in der fetalen Periode

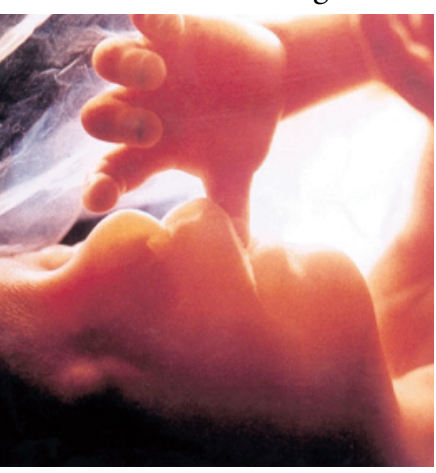
könnten die Entwicklung des Immun- und respiratorischen Systems so beeinflussen, dass sie das Allergie-Risiko erhöhen. Epidemiologen aus Oslo prüften in einer prospektiven Studie mit 2.531 Kindern, ob Komplikationen in der Schwangerschaft häufiger Asthma und allergische Rhinitis zur Folge haben. Sie dokumentierten den Gesundheitszustand und Umweltfaktoren bei der Geburt, nach 6, 12, 18 und 24 Monaten sowie im Alter von vier Jahren.

Tatsächlich fand sich eine Beziehung zwischen Gebärmutter-assoziierten Komplikationen (antepartale Blutungen, vorzeitige Wehen, Plazentainsuffizienz und ungenügendes Gebärmutterwachstum) und einem 3 fach erhöhten Risiko für Asthma und allergische Rhinitis - unabhängig von der Familienanamnese. $c l$

Nafstad P et al. J Allergy Clin Immunol

2000; 106: 867-73.

\section{Patch-Test vor Reimplantation}

oronar-Stents retten Leben - doch $10 \%$ der Patienten erleiden eine „InStent"-Restenose. Eppendorfer Kardiound Dermatologen prüften in einem Gemeinschaftsprojekt ihre Vermutung, dass eine kontaktallergische Reaktion auf freigesetztes Stentmaterial die Restenose fördert. Denn die meisten Stents bestehen aus 316L Edelstahl, der stark sensibilisierende Metalle enthält, u.a. Nickel, Chrom-Salze und Molybdän.

Bei 131 KHK-Patienten, die sechs Monate nach Stentimplantation klinische Zeichen einer Restenose boten, führten die Dermatologen Patch-Tests

\section{Wuchernde Allergie}

든 in 22-jähriger Student mit Allergieanamnese suchte die Nothilfe wegen einer Visusminderung auf. Bei der Eversion des Oberlides erkannte man zwei große polypoide konjunktivale Läsionen. Diese wurden operativ entfernt. Die histologische Untersuchung ergab Hinweise für allergische Genese.

Pflastersteinartige Proliferationen der tarsalen Bindehaut, die zu einem erheblichen Fremdkörpergefühl führen können, sind typisch für die Konjunctivitis vernalis. Mitteleuropäische Patienten haben diese Läsion meistens im Bereich der tarsalen Bindehaut. Bei Pati- durch. Sieben der Patienten entwickelten eine Spät-Reaktion auf Nickel, vier reagierten positiv auf Molybdän. Die Angiographie zeigte bei allen zehn Patienten mit positivem Patch-Test eine Restenose (10 von 89 Restenose-Patienten vs. 0 von 42 Patienten ohne Stent-Stenose; $\mathrm{p}=0,03$ ). Dennoch hatten $89 \%$ der Restenose-Patienten einen negativen Patch-Test. Die Autoren empfehlen, eine Metall-Sensibilisierung bei Patienten mit In-Stent-Restenose auszuschließen, bevor sie einen neuen Stent erhalten. $\quad c l$

Köster R et al. Lancet 2000; 356: 1895-7.

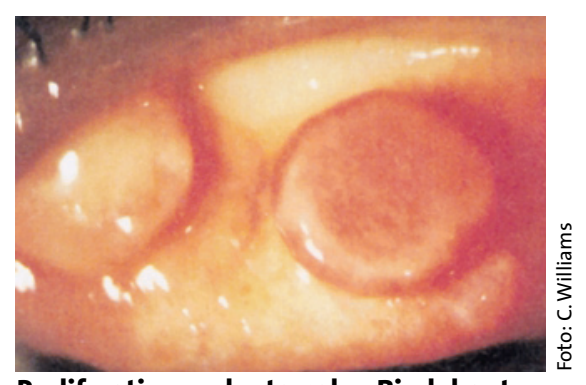

Proliferationen der tarsalen Bindehaut

enten aus dem Mittelmeerraum kommt es dagegen mehr zu Läsionen im Bereich des Limbus cornea, welche die Hornhaut gefährden können. In der Bindehaut sind massenhaft eosinophile Granulozyten zu finden, was auf die allergische Genese hinweist.

H. S. Füeßl

\section{AE auf Chromosom 3}

Corscher der Charité-Kinderklinik der Humboldt-Universität zu Berlin und des Max-Delbrück-Centrums für Molekulare Medizin (MDC) BerlinBuch haben in einer europaweiten Studie erstmals ein Gen für das atopische Ekzem (AE) auf Chromosom 3 lokalisiert.

An der vom Forschungsministerium und vom Deutschen Humangenomprojekt geförderten Studie beteiligten sich fast 200 Familien mit über 800 Mitgliedern aus Deutschland, Italien, Schweden und den Niederlanden. Jede der Familien hatte mindestens zwei Kinder, die vor dem zweiten Geburtstag an AE erkrankt waren. Dreiviertel der 421 Kinder hatten zusätzliche Allergien. Nach systematischer Durchmusterung aller Chromosomen zeigte sich, dass das $\mathrm{AE}$ und Allergien besonders häufig mit einem bestimmten Abschnitt des Chromosoms 3 vererbt wurden. Zur Allergie kam es vor allem dann, wenn dieser Genort von der Mutter stammte, während die Vererbung vom Vater kaum Wirkung auf die Allergieentwicklung hatte. Weitere Untersuchungen sollen das Gen genau identifizieren. Die Forscher bitten daher Familien mit mindestens zwei an AE erkrankten Kindern, an der Studie Teil zu nehmen. $c l$

Lee Y-A et al. Nature Genetics 2000; 26: 470-3.

\section{Risikoeinkauf}

achforschungen der VerbraucherZentrale Hamburg e.V. ergaben, dass drei von acht untersuchten Schokoladen und Keks-Sorten mit Haselnuss, eine mit Erdnusseiweiß verunreinigt waren. In fünf Chips-Proben fanden sie Bestandteile von Weizeneiweiß. Keiner der Produkte war entsprechend gekennzeichnet.

Daher appellieren die VerbraucherZentrale und der Deutsche Allergie- und Asthmabund (DAAB) an den Gesetzgeber, die lückenlose Kennzeichnung aller Inhaltsstoffe gesetzlich zu verankern. Von den Herstellern fordern sie eine bessere Kontolle der Produktionsabläufe. $c l$ 